\title{
Rayleigh-Taylor Instability of a Compressible Plasma with Finite Larmor Radius Effects
}

\author{
P. D. Ariel \\ Institut für Mechanik, Universität Hannover, Hannover, Germany*
}

Z. Naturforsch. 48a, 844-850 (1993); received April 14, 1993

The Rayleigh-Taylor instability of a compressible plasma in the presence of a horizontal magnetic field is investigated, taking into account the effects of finite Larmor radius. Only transverse perturbations are considered. The problem is shown to be characterized by a variational principle. Using it, the dispersion relation is obtained for a plasma layer of finite thickness and having an exponentially varying density. It is found that the finite Larmor radius effects can thoroughly stabilize unstable configurations. For configurations which are not completely stabilized, the compressibility stabilizes some of the disturbances which are unstable for an incompressible plasma.

The Rayleigh-Taylor instability (RTI) derives its character from adverse density gradients. It figures prominently in astrophysical and laboratory phenomena. In most of these situations a magnetic field plays an important part. Chandrasekhar [1] has described in detail the various aspects of RTI in hydromagnetics when the direction of the magnetic field is either horizontal or vertical. For a general orientation of the magnetic field, the stability criterion has been analyzed by Ariel and Aggarwala [2].

There are several astrophysical situations in which the finite Larmor radius (FLR), which is usually neglected, cannot be ignored. Rosenbluth et al. [3] showed that the inclusion of the FLR effects can stabilize several "weakly" unstable systems such as mirror machines, slowly rotating plasmas, large aspect ratio torii, etc. They used kinetic equations to cater for the FLR effects. Roberts and Taylor [4] demonstrated that the FLR effects can be effectively included in the fluid equations in the form of magnetic viscosity terms. These modified equations have been extensively used for further investigation of stability problems with the FLR effects. Thus Singh and Hans [5] showed that for the RTI of two superposed plasmas all transverse perturbations corresponding to wave numbers beyond a critical value are stabilized by the magnetic field due to the inclusion of the FLR effects. For the other

\footnotetext{
* On leave of absence from Moi University, P.O. Box 3900, Eldoret, Kenya.
}

Reprint requests to Prof. Dr. P. D. Ariel, Moi University, P.O. Box 3900, Eldoret, Kenya. important configuration, namely a plasma with an exponentially varying density in the vertical direction, it has been demonstrated by Ariel and Bhatia [6] that FLR effects can completely stabilize certain unstable configurations. In fact, the larger the gradient, the more the configuration is stabilized. For a rotating stratified plasma a similar conclusion holds (Ariel and Bhatia [7]).

Studies of the effects of FLR on other instabilities have been undertaken during the last two decades. One can mention thermal and thermosolutal instability (Sharma et al. [8], Sharma and Sharma [9], Sharma and Rani [10], Sharma and Misra [11]) and gravitational instability when a conducting matter is surrounded by a non-conducting matter (Ariel [12]). In all these investigations the perturbations are chosen transverse to the horizontal magnetic field. For longitudinal perturbations the stability influence is not so pronounced. In fact, as recently shown by Ariel [13], for RTI the basic stability criterion remains unaffected; only the rate at which the instability overtakes the system is slowed down.

Since most plasmas occurring in nature are compressible, a more realistic study must allow their compressibility. The literature on the RTI of a compressible plasma is rather scarce, no doubt because of the complexities arising on account of the compressibility. Srivastava [14] for the first time attempted to investigate the effects of FLR on the RTI of a compressible plasma with exponentially varying density in the vertical direction. He considered both perturbations: longitudinal and transverse. During the course of his analysis Srivastava [14] made a number of simplifica-

0932-0784 / 93 / 0800-0844 $\$ 01.30 / 0$ - Please order a reprint rather than making your own copy. 
tions and approximations which may not be justified or even necessary.

In the present paper we re-examine the problem of Srivastava [14], i.e. we investigate the stability of a layer of compressible plasma stratified in the vertical direction in the presence of a horizontal magnetic field, taking into account the finiteness of the Larmor radius. We have confined ourselves to only transverse perturbations. Our objective is rather modest. In line with our earlier work (Ariel and Bhatia [6]) we intend to investigate if there are certain unstable configurations that can be stabilized by the FLR effects. This aspect, we feel, is more important than finding out if the rate at which the configuration departs from equilibrium is slowed down by the inclusion of the FLR effects. It was ignored by Srivastava [14].

\section{Perturbation Equations}

We start with general equations. Consider a plasma layer of density $\varrho(z)$ confined between the planes $z=0$ and $z=d$, where the $z$-axis is taken in the vertical direction. The layer is stratified in a gravitational field $\boldsymbol{g}(0,0,-g)$ and is pervaded by a uniform magnetic field $\boldsymbol{H}(H, 0,0)$. It is assumed that the plasma is compressible, inviscid and perfectly conducting.

The linearized perturbation equations resulting from giving a disturbance to the configuration that produces a velocity field $u(u, v, w)$ are

$$
\begin{aligned}
& \varrho \frac{\partial \boldsymbol{u}}{\partial t}=-\nabla \cdot \delta \Pi+\frac{1}{4 \pi} \nabla \times(\boldsymbol{h} \times \boldsymbol{H})+\boldsymbol{g} \delta \varrho, \\
& \frac{\partial}{\partial t} \delta \varrho=-\varrho \nabla \cdot \boldsymbol{u}-\boldsymbol{u} \cdot \nabla \varrho \\
& \frac{\partial}{\partial t} \delta p+\boldsymbol{u} \cdot \nabla p=c^{2}\left(\frac{\partial}{\partial t} \delta \varrho+\boldsymbol{u} \cdot \nabla \varrho\right), \\
& \frac{\partial \boldsymbol{h}}{\partial t}=\nabla \times(\boldsymbol{u} \times \boldsymbol{H}),
\end{aligned}
$$

and

$$
\boldsymbol{\nabla} \cdot \boldsymbol{h}=0,
$$

where $\delta \varrho, \boldsymbol{h}\left(h_{x}, h_{y}, h_{z}\right)$ are the perturbations in $\varrho$ and $\boldsymbol{H}$, respectively, $c$ is the velocity of sound in the medium and $\delta \Pi$ represents the perturbation in the stress tensor $\Pi$, which because of the inclusion of the
FLR effects takes the form (Roberts and Taylor [4])

$$
\begin{aligned}
& \Pi_{x x}=p, \\
& \Pi_{y y}=p-\varrho v\left(\frac{\partial v}{\partial z}+\frac{\partial w}{\partial y}\right), \\
& \Pi_{z z}=p+\varrho v\left(\frac{\partial v}{\partial z}+\frac{\partial w}{\partial y}\right), \\
& \Pi_{x y}=\Pi_{y x}=-2 \varrho v\left(\frac{\partial w}{\partial x}+\frac{\partial u}{\partial z}\right), \\
& \Pi_{x z}=\Pi_{z x}=2 \varrho v\left(\frac{\partial u}{\partial y}+\frac{\partial v}{\partial x}\right), \\
& \Pi_{y z}=\Pi_{z y}=\varrho v\left(\frac{\partial v}{\partial y}-\frac{\partial w}{\partial z}\right) .
\end{aligned}
$$

In (6), $p$ denotes the isotropic pressure and $v=a^{2} \Omega / 4$, $a$ being the ion Larmor radius and $\Omega$ the ion gyro-frequency. In (3) $\delta p$ is the perturbation in $p$.

It may be remarked here that Roberts and Taylor [4] derived the above expressions for the componentsof $\Pi$ assuming that the magnetic field is constant. Srivastava [14], on the other hand, has used the same expressions even for a variable magnetic field. It is true that later in his analysis Srivastava goes to make the assumption that the gradients of density and magnetic field are small, in which case his results may probably be still valid in the zeroth order approximation. However, taking the density gradient small, rules out the possibility of examining the stabilizing role of the FLR effects on those configurations which are most affected by these effects (see Ariel and Bhatia [6]).

We seek the solution of the perturbation equations in terms of normal modes in which the $y$ and $t$ dependence is given by

$$
\exp (i k y+n t),
$$

where $k$ is the wave number and $n$ the rate at which the system departs from equilibrium. Note that we are only considering transverse perturbations.

Equations (1)-(6) take the form

$$
\begin{aligned}
n \varrho u= & 2 i k v D \varrho u \\
n \varrho v= & -i k \delta p+\varrho v\left(D^{2}-k^{2}\right) w \\
& -v D \varrho(i k v-D w)-(H / 4 \pi) i k h_{x}, \\
n \varrho w= & -D \delta p-\varrho v\left(D^{2}-k^{2}\right) v \\
& -v D \varrho(D v+i k w)-g \delta \varrho-(H / 4 \pi) D h_{x}, \\
n \delta \varrho= & -\varrho \nabla \cdot u-w D \varrho
\end{aligned}
$$


$n \delta p=g \varrho w-c^{2} \varrho \nabla \cdot \boldsymbol{u}$,

$n h_{x}=-H \nabla \cdot u$,

$\boldsymbol{\nabla} \cdot \boldsymbol{u}=i k v+D w$

where $D$ stands for $\mathrm{d} / \mathrm{d} z$.

One can eliminate various quantities from (8)-(14) to arrive at the following pair of equations in $w$ and $\boldsymbol{\nabla} \cdot \boldsymbol{u}$ :

$$
\begin{aligned}
& n\left[D(\varrho D w)-k^{2} \varrho w\right]+\left(g k^{2} / n\right) D \varrho w \\
& \quad+2 i v k\left[D(D \varrho D w]-k^{2}(D \varrho) w-n D(\varrho \nabla \cdot u)\right. \\
& \quad+\left(g k^{2} / n\right) \varrho \nabla \cdot u \\
& \quad+i v k\left[\varrho\left(D^{2}-k^{2}\right) \nabla \cdot u-D^{2} \varrho \nabla \cdot u\right]=0
\end{aligned}
$$

and

$$
\begin{aligned}
{[\varrho n+i v k D \varrho} & \left.+\left(k^{2} / n\right)\left(\varrho c^{2}+H^{2} / 4 \pi\right)\right] \nabla \cdot u \\
=\varrho n D w & +\left(g k^{2} / n\right) \varrho w+i v k \varrho\left(D^{2}-k^{2}\right) w \\
& +2 i v k D \varrho D w .
\end{aligned}
$$

Of course, the value of $\boldsymbol{\nabla} \cdot \boldsymbol{u}$ can be substituted from (16) into (15) to obtain a single differential equation in $w$. For the present, however, we shall prefer to deal with the pair of equations. Also, we find it convenient to introduce $\omega$, a measure of the frequency of oscillations of the disturbance, defined by

$$
n=i \omega,
$$

in which case (15) and (16) can be rewritten as

$$
\begin{aligned}
& \omega\left[D(\varrho D w)-k^{2} \varrho w\right]-\frac{g k^{2} D \varrho}{\omega} w \\
& +2 v k\left[D(D \varrho D w)-k^{2}(D \varrho) w\right]-\omega D(\varrho \nabla \cdot u) \\
& -\frac{g k^{2} \varrho}{\omega} \nabla \cdot u+v k\left[\varrho\left(D^{2}-k^{2}\right) \nabla \cdot u-D^{2} \varrho \nabla \cdot u\right]=0,
\end{aligned}
$$

and

$$
\begin{aligned}
& {\left[\varrho \omega+v k D \varrho-\frac{k^{2}}{\omega}\left(\varrho c^{2}+\frac{H^{2}}{4 \pi}\right)\right] \nabla \cdot u} \\
& =\varrho \omega D w-\frac{g k^{2} \varrho}{\omega} w+v k \varrho\left(D^{2}-k^{2}\right) w+2 v k D \varrho D w .
\end{aligned}
$$

\section{Boundary Conditions}

The boundary conditions on $w$ are

$$
w(0)=0, \quad w(d)=0,
$$

which imply that vertical motion is prohibited at the boundaries.

Following Hosking and Harinoff [15], we shall assume

$$
\boldsymbol{\nabla} \cdot \boldsymbol{u}(0)=0, \quad \boldsymbol{\nabla} \cdot \boldsymbol{u}(d)=0 .
$$

This boundary condition is only an approximation and we shall be requiring it only to establish the existence of the variational formulation for the problem at hand.

\section{A Variational Formulation}

We will now show that the present problem is characterized by a variational principle. For this we multiply (18) by $w$ and integrate across the vertical extent of the fluid to obtain

$$
\begin{gathered}
\omega \int_{0}^{d} \varrho\left[(D w)^{2}+k^{2} w^{2}\right] \mathrm{d} z+\frac{g k^{2}}{\omega} \int_{0}^{d} D \varrho w^{2} \mathrm{~d} z \\
+2 v k \int_{0}^{d} D \varrho\left[(D w)^{2}+k^{2} w^{2}\right] \mathrm{d} z \\
+\int_{0}^{d}\left\{\omega D(\varrho \nabla \cdot \boldsymbol{u})-v k\left[\varrho\left(D^{2}-k^{2}\right) \nabla \cdot \boldsymbol{u}\right.\right. \\
\left.\left.-D^{2} \varrho \nabla \cdot \boldsymbol{u}\right]+\frac{g \varrho k^{2}}{\omega} \nabla \cdot \boldsymbol{u}\right\} \mathrm{d} z=0,
\end{gathered}
$$

in which use has been made of the boundary condition (20) on $w$, for example

$\int_{0}^{d} D(D \varrho D w) \mathrm{wd} z=\left.D \varrho w D w\right|_{0} ^{d}-\int_{0}^{d} D \varrho(D w)^{2} \mathrm{~d} z$.

The integrated part becomes zero on account of the vanishing of $w$ at the boundary.

The $\boldsymbol{\nabla} \cdot \boldsymbol{u}$ term in (22) can be written as

$$
\begin{gathered}
\int_{0}^{d}\left\{-\varrho \omega D w-v k\left[D^{2}(\varrho w)-\varrho k^{2} w-D^{2} \varrho w\right]\right. \\
\left.+\frac{g \varrho k^{2}}{\omega} w\right\} \nabla \cdot \boldsymbol{u} \mathrm{d} z,
\end{gathered}
$$

where we have again integrated a few terms by parts. The integrated parts vanish on account of the boundary condition (20) or (21). The above term can be simplified to

$$
\begin{aligned}
-\int_{0}^{d}\left\{\varrho \omega D w+\varrho v k\left(D^{2}-k\right) w\right. \\
\left.\quad+2 D \varrho v k D w-\frac{g \varrho k^{2}}{\omega} w\right\} \nabla \cdot \boldsymbol{u} \mathrm{d} z .
\end{aligned}
$$


Now use is made of (19) for substituting for $w$-terms in (25) and inserting the result in (22). One finally obtains

$$
\begin{aligned}
& \omega \int_{0}^{d} \varrho\left[(D w)^{2}+k^{2} w^{2}\right] \mathrm{d} z+\frac{g k^{2}}{\omega} \int_{0}^{d} D \varrho w^{2} \mathrm{~d} z \\
& +2 v k \int_{0}^{d} D \varrho\left[(D w)^{2}+k^{2} w^{2}\right] \mathrm{d} z \\
& -\int_{0}^{d}\left[\varrho \omega+D \varrho v k-\frac{k^{2}}{\omega}\left(\varrho c^{2}+\frac{H^{2}}{4 \pi}\right)\right](\nabla \cdot u)^{2} \mathrm{~d} z=0 .
\end{aligned}
$$

Equation (26) is the required variational formulation of the problem. We now show that, if the first order variations $\delta w$ and $\delta \boldsymbol{\nabla} \cdot \boldsymbol{u}$ are made in $w$ and $\boldsymbol{\nabla} \cdot \boldsymbol{u}$, respectively, which are consistent with the boundary conditions (20) and (21), then $\delta \omega$ is zero to the first order or $\omega$ is stationary. The implementation of the suggested variations in (26) yields to the first order

$$
\begin{aligned}
\frac{1}{2} \delta \omega \int_{0}^{d}\{\varrho & {\left[(D w)^{2}+k^{2} w^{2}\right]-\frac{g k^{2} D \varrho}{\omega^{2}} w^{2} } \\
- & {\left.\left[\varrho+\frac{k^{2}}{\omega^{2}}\left(\varrho c^{2}+\frac{H^{2}}{4 \pi}\right)\right](\nabla \cdot u)^{2}\right\} \mathrm{d} z }
\end{aligned}
$$

$+\int_{0}^{d} \varrho\left[D w D \delta w+k^{2} w \delta w\right] \mathrm{d} z+\frac{g k^{2}}{\omega} \int_{0}^{d} D \varrho w \delta w \mathrm{~d} z$

$+2 v k \int_{0}^{d} D \varrho\left[D w D \delta w+k^{2} w \delta w\right] \mathrm{d} z$

$-\int_{0}^{d}\left[\varrho \omega+D \varrho v k-\frac{k^{2}}{\omega}\left(\varrho c^{2}+\frac{H^{2}}{4 \pi}\right)\right] \nabla \cdot u \delta \nabla \cdot u \mathrm{~d} z=0$.

The variations $\delta w$ and $\delta \nabla \cdot u$ are not independent. In fact, they are related through (19), the relation being

$$
\begin{array}{r}
{\left[\varrho \omega+D \varrho v k-\frac{k^{2}}{\omega}\left(\varrho c^{2}+\frac{H^{2}}{4 \pi}\right)\right] \delta \nabla \cdot u} \\
=\varrho \omega D \delta w-v \varrho k\left(D^{2}-k^{2}\right) \delta w-2 D \varrho v k D \delta w \\
+\frac{g k^{2} \varrho}{\omega} \delta w-\left\{\left[\varrho+\frac{k^{2}}{\omega^{2}}\left(\varrho c^{2}+\frac{H^{2}}{4 \pi}\right)\right] \nabla \cdot \boldsymbol{u}\right. \\
\left.\quad-\varrho D w-\frac{g k^{2} \varrho}{\omega^{2}} w\right\} \delta w .
\end{array}
$$

The substitution of $\delta \boldsymbol{\nabla} \cdot \boldsymbol{u}$ from (28) into (27) and a few integrations by parts, for which use is made of the appropriate boundary conditions on $w, \boldsymbol{\nabla} \cdot \boldsymbol{u}$ or $\delta w$ and $\delta \nabla \cdot u$, yields the following equation for $\delta \omega$ :

$$
\begin{gathered}
\frac{1}{2} \delta \omega \int_{0}^{d}\left\{\varrho\left[(\nabla \cdot u-D w)^{2}+k^{2} w^{2}\right]+\frac{k^{2}}{\omega^{2}}\left(\varrho c^{2}+\frac{H^{2}}{4 \pi}\right)(\nabla \cdot u)^{2}\right. \\
\left.-\frac{2 g \varrho k^{2}}{\omega^{2}} w(\nabla \cdot u-D w)\right\} \mathrm{d} z \\
=\int_{0}^{d}\left\{\omega\left[D(\varrho D w)-k^{2} \varrho w\right]-\frac{g k^{2} D \varrho}{\omega} w\right. \\
+2 v k\left[D(D \varrho D w)-k^{2} D \varrho w\right]-\omega D(\varrho \nabla \cdot u) \\
\left.-\frac{g k^{2} \varrho}{\omega} \nabla \cdot u+v k\left[\varrho\left(D^{2}-k^{2}\right) \nabla \cdot u-D^{2} \varrho \nabla \cdot u\right]\right\} \delta w \mathrm{~d} z .
\end{gathered}
$$

From (29) it is clear that a necessary and sufficient condition for $\delta \omega$ to vanish in the first order approximation is that $w$ and $\boldsymbol{\nabla} \cdot \boldsymbol{u}$ satisfy the eigen-value problem (18)-(21). Hence a variational principle for finding an approximate solution is available. Note that its existence is valid for any general density distribution.

\section{The Case of Exponentially Varying Density}

In the present section we consider the case of a plasma layer confined between planes $z=0$ and $z=d$, in which in the undisturbed state the density is given by

$$
\varrho=\varrho_{0} e^{\beta z},
$$

$\varrho_{0}$ being the density at the lower boundary.

One can substitute for $\boldsymbol{\nabla} \cdot \boldsymbol{u}$ from (19) into (26) to obtain the equivalent variational formulation

$$
\begin{aligned}
& \omega^{2} \int_{0}^{d} \varrho\left[(D w)^{2}+k^{2} w^{2}\right] \mathrm{d} z+g k^{2} \int_{0}^{d} D \varrho w^{2} \mathrm{~d} z \\
& +2 v k \omega \int_{0}^{d} D \varrho\left[(D w)^{2}+k^{2} w^{2}\right] \mathrm{d} z \\
& -\int_{0}^{d} \frac{\left[\varrho \omega^{2} D w+\varrho v k \omega\left(D^{2}-k^{2}\right) w+2 D \varrho v k \omega D w-g k^{2} \varrho w\right]^{2}}{\varrho \omega^{2}+D \varrho v k \omega-k^{2}\left(\varrho c^{2}+H^{2} / 4 \pi\right)} \mathrm{d} z=0,
\end{aligned}
$$

which is more useful as it contains only the variable $w$.

Now, in order to obtain an approximate solution, any trial function can be chosen for $w$ which satisfies the boundary conditions of the problem. Our choice is the same value of $w$ which is the exact solution for the case of the incompressible plasma. It is given by (Ariel and Bhatia [6])

$$
w=W \exp \left\{-\frac{1}{2} \beta z\right\} \sin l z,
$$


where

$$
l=\pi s / \mathrm{d},
$$

$s$ being an integer.

In principle, the value of $w$ can be substituted from (32) into (31) in order to obtain the required dispersion relation between $k$ and $\omega$. In practice, the evaluation of the last integral is not easy. In keeping with the spirit of an approximate solution it seems reasonable to approximate the integral also and evaluate it by using some integration rules.

Now the use of any integration rule results in (Isaacson and Keller [15])

$$
I(f) \equiv \int_{a}^{b} f(x) \mathrm{d} x=\sum_{1}^{N} \alpha_{i} f\left(x_{i}\right),
$$

where $N$ is a finite integer, $x_{i} \in[a, b]$ and $\alpha_{i}$ are the corresponding weights for the nodes $x_{i}$.

If the value of $w$ is substituted from (32) into (31) and the discretization is performed using the general integration rule (34), this will yield a dispersion relation which is a polynomial of degree $2 N+2$ in $\omega$ with real coefficients. For a polynomial with real coefficients, since the complex roots occur in pairs, we can conclude that for stability a necessary and sufficient condition is that $\omega$ is real. For if $\omega$ is complex, corresponding to the root with the negative imaginary part, the real part of $n$ given by (17) will be positive. This implies instability of the system - in fact, overstability because of the oscillatory nature of the motion.

It is evident that a too large value of $N$ would give rise to a rather unwieldy dispersion relation. Ideally, one would like to choose $N=1$. This can be accomplished by invoking the mean value theorem of integral calculus. Thus, taking $\omega$ to be real for stability, the last term in (31) can be written as

$$
\begin{gathered}
-\left[\omega^{2}+\left(D \varrho^{*} / \varrho^{*}\right) v k \omega-k^{2}\left(c^{* 2}+H^{2} / 4 \pi \varrho^{*}\right)\right]^{-1} \\
\cdot \int_{0}^{d} \varrho\left[\omega^{2} D w+v k \omega\left(D^{2}-k^{2}\right) w\right. \\
\left.+2(D \varrho / \varrho) v k \omega D w-g k^{2} w\right]^{2} \mathrm{~d} z,
\end{gathered}
$$

where a starred quantity denotes the value of that quantity at some interior point in the domain $(0, d)$. Under the Boussinesq approximation, these quantities can be taken to correspond to the lower boundary, but that will restrict us to smaller density gradients which, as remarked earlier, would not allow us to see the effects of FLR in the proper perspective.

The values of $\varrho$ and $w$ are now substituted from (30) and (32), respectively, in (31), and the integrals are evaluated after use is made of (35). As a result the following dispersion relation is obtained:

$$
\begin{aligned}
& \omega^{2}-2 v \beta k \omega-\frac{g \beta k^{2}}{l^{2}+k^{2}+\frac{1}{4} \beta^{2}} \\
& -\frac{l^{2}\left(\omega^{2}+v \beta k \omega\right)^{2}+\left[g k^{2}+\frac{1}{2} \omega^{2} \beta+v k \omega\left(l^{2}+k^{2}+\frac{3}{4} \beta^{2}\right)\right]^{2}}{\left(l^{2}+k^{2}+\frac{1}{4} \beta^{2}\right)\left[\omega^{2}+v \beta k \omega-k^{2}\left(c^{* 2}+V^{* 2}\right)\right]}=0,
\end{aligned}
$$

where

$$
V^{*}=\sqrt{\frac{H^{2}}{4 \pi \varrho^{*}}}
$$

is a characteristic Alfven velocity in the medium.

It is easy to see that for an incompressible plasma $\left(c^{*} \rightarrow \infty\right),(36)$ reduces to the corresponding dispersion relation obtained by Ariel and Bhatia [6]. In the absence of FLR effects $(v=0),(36)$ is essentially the same as that obtained by Talwar [16] for the RTI of an compressible fluid in the presence of a horizontal magnetic field for transverse perturbations. It is also worth noting that, even though we are considering transverse perturbations, unlike the case of incompressible plasma, the magnetic field has still a direct influence on the dispersion relation because of the presence of the term involving $V^{*}$.

Equation (36) can be expanded and written as a biquadratic in $\omega$ as under

$$
\begin{aligned}
\omega^{4} & +2 v \beta k \omega^{3} \\
& -\left\{\left(\alpha^{2}+k^{2}\right)\left(c^{* 2}+V^{* 2}\right)+v^{2}\left[\left(\alpha^{2}+k^{2}\right)^{2}-\beta^{2} k^{2}\right]\right\} \omega^{2} \\
& -2 v k \omega\left(\alpha^{2}+k^{2}\right)\left[\beta\left(c^{* 2}+V^{* 2}\right)+g\right] \\
& -g k^{2}\left[\beta\left(c^{* 2}+V^{* 2}\right)+g\right]=0
\end{aligned}
$$

where

$$
\alpha^{2}=l^{2}+\frac{1}{4} \beta^{2} .
$$

It will be found convenient in the ensuing analysis to measure the quantities $k$ and $\omega$ in units of $\alpha \mathrm{cm}^{-1}$ and $(g \beta)^{1 / 2} \mathrm{sec}^{-1}$. This allows (38) to be expressed in the non-dimensional form

$$
\begin{aligned}
\omega^{4}+ & 2 \sqrt{G} k \omega^{3} \\
& -\left\{Q\left(1+k^{2}\right)+G\left[a\left(1+k^{2}\right)^{2}-k^{2}\right]\right\} \omega^{2} \\
& -2 \sqrt{G} k\left(1+k^{2}\right)(Q+a) \omega-k^{2}(Q+a)=0,
\end{aligned}
$$

where

$$
\begin{aligned}
& G=\frac{v^{2} \alpha^{2} \beta}{g}, \\
& a=\frac{\alpha^{2}}{\beta^{2}},
\end{aligned}
$$


and

$$
Q=\frac{\alpha^{2}\left(c^{* 2}+V^{* 2}\right)}{g \beta}
$$

are dimensionless numbers. $G$ is a measure of the magnetic viscosity, $a$ determines the density gradient and $Q$ is a number characterizing the joint effect of compressibility and the magnetic field in terms of the buoyancy forces.

\section{Analysis of the Dispersion Relation}

Equation (40), being a biquadratic, admits four roots which are either all real or at least one pair complex. For stability, we require all the four roots to be real. Now the biquadratic

$$
a x^{4}+b x^{3}+c x^{2}+d x+e=0
$$

has all its roots real or two roots real and the remaining roots a pair of complex conjugates according to

$$
\Delta \equiv I^{3}-27 J^{2} \gtrless 0,
$$

where

$$
\begin{aligned}
& I=a e-4 b d+3 c^{2}, \\
& J=a c e+2 b c d-a d^{2}-e b^{2}-c^{3} .
\end{aligned}
$$

A substitution is made of the coefficients of various terms in (40) into (46) to calculate $I$ and $J$. These values, in turn, are inserted in (45) to calculate $\Delta$. The change in the sign of $\Delta$ separates the stable and unstable configurations. Evidently $\Delta$ is a function of $k$. It also involves the parameters $G, a$, and $Q$.

For a given set of parameters $G, a$, and $Q, k<0$ (at $k=0, \Delta$ is zero). For small values of $k$, it can be verified that

$\Delta=-\frac{1}{16} Q(Q+G a)^{3}(Q+a)(1-G) k^{2}, \quad k \rightarrow 0$.

Thus, if $G>1, \Delta$ is positive for small values of $k$. In fact, when $G>1$ it is found that $\Delta$ is positive for all values of $k$ irrespective of the values of $Q$ and $a$. Hence the configuration characterized by $G>1$ is completely stabilized by the finite Larmor radius effect, and this result does not depend on the compressibility of the plasma. This conclusion is identical to the one derived by Ariel and Bhatia [6] for incompressible plasmas.

In order to see the effects of compressibility on the onset of instability let us, therefore, restrict ourselves

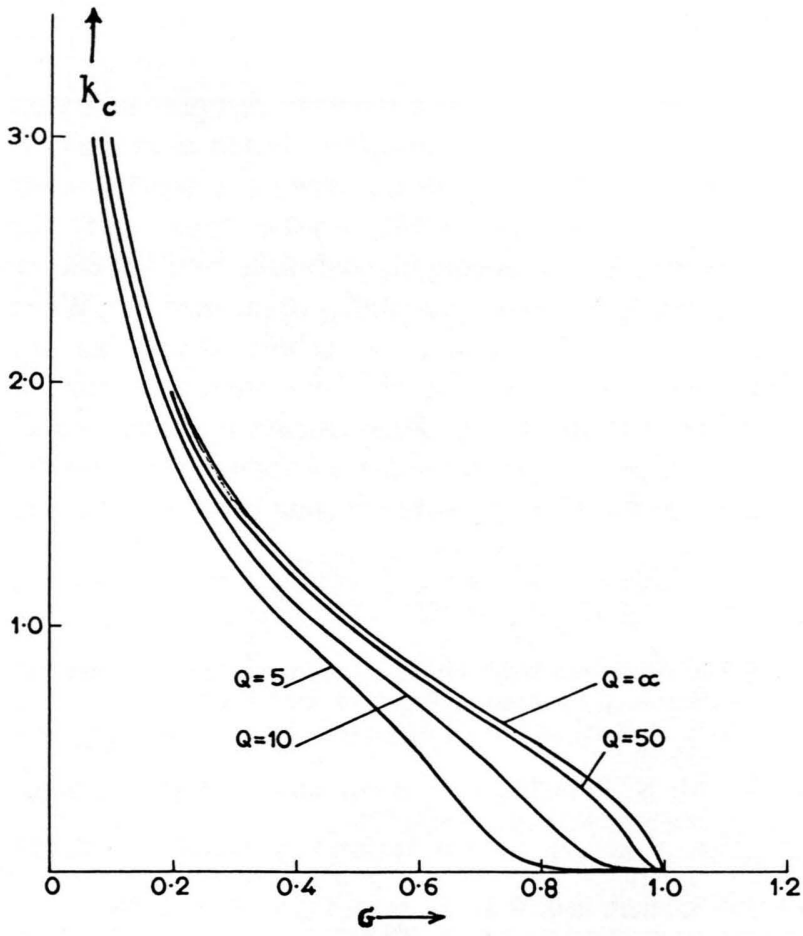

Fig. 1. Illustrating the variation of $k_{\mathrm{c}}$, the critical wave number, with $G$, the dimensionless measure of the FLR effects, for $a=1$ and various values of $Q$, a measure of the joint effects of compressibility and magnetic field in terms of buoyancy forces.

to the case $G<1$. Now, for small values of $k, \Delta$ is negative, implying overstability. For large values of $k$, the behavior of $\Delta$ is

$$
\Delta=\frac{1}{16} G^{4} a^{3} Q(Q+a) k^{18}, \quad k \rightarrow \infty .
$$

Since $\Delta>0$, the configuration is stabilized for large values of $k$. From the foregoing it appears that there exists a critical value of $k$, say $k_{\mathrm{c}}$, where the configuration goes through the transition from being overstable to stable. This was confirmed numerically by computing the value of $\Delta$ as a function of $k$ for $G<1$ and various values of $Q$ and $a$.

In Fig. $1, k_{\mathrm{c}}$ is plotted against the $G$ for $a=1$ and various values of $Q$. The region $k>k_{\mathrm{c}}$ is stabilized by the FLR effects, and the region $k<k_{\mathrm{c}}$ remains overstable. As the value of $Q$ is decreased, one may note from the figure, the value of $k_{\mathrm{c}}$ also decreases. Thus the compressibility of the plasma stabilizes certain modes which are otherwise unstable. 


\section{Conclusions}

We have examined the effects of compressibility on the RTI, taking into account the finiteness of the Larmor radius. It is shown that when the dimensionless parameter $G$, defined by (41), is greater than unity, the configuration is thoroughly stabilized by FLR effects, regardless of the compressibility of the medium. When $G$ is smaller than unity, overstability takes place for values of $k$, the wave number of disturbance, smaller than a critical value $k_{\mathrm{c}}$. This critical value decreases as the compressibility of the medium is increased, implying that for a compressible plasma certain modes are

[1] S. Chandrasekhar, Hydrodynamic and Hydromagnetic Stability. Clarendon Press, Oxford 1962.

[2] P. D. Ariel and B. D. Aggarwala, Can. J. Phys. 57, 1094 (1979).

[3] M. N. Rosenbluth, N. Krall, and N. Rostoker, Nucl. Fusion Suppl. 1, 143 (1963).

[4] K. V. Roberts and J. B. Taylor, Phys. Rev. Letters 8, 197 (1962).

[5] S. Singh and H. Hans, Nucl. Fusion 6, 6 (1966).

[6] P. D. Ariel and P. K. Bhatia, Can. J. Phys. 47, 2235 (1969).

[7] P. D. Ariel and P. K. Bhatia, Nucl. Fusion 10, 141 (1970).

[8] R. C. Sharma, R. Nyland, and K. P. Thakur, Physica 112 B, C, 341 (1981). stabilized which were unstable for incompressible plasma.

\section{Acknowledgements}

The author wishes to thank DAAD (Deutscher Akademischer Austauschdienst) for the award of a visiting fellowship, and Moi University for providing the travel funds. He is also grateful to the referee for drawing attention to a questionable argument used in the earlier version of the paper to derive the dispersion relation.

[9] R. C. Sharma and K. N. Sharma, Phys. Fluids 24, 2242 (1981).

[10] R. C. Sharma and N. Rani, Z. Naturforsch. 41 a, 724 (1986).

[11] R. C. Sharma and J. N. Misra, Astrophys. Space Sci. 126, 223 (1986).

[12] P. D. Ariel, Astrophys. Space Sci. 141, 141 (1988).

[13] P. D. Ariel, Astrophys. Space Sci. 196, 153 (1992).

[14] K. M. Srivastava, Z. Naturforsch. 29a, 518 (1974).

[15] E. Isaacson and H. B. Keller, Analysis of Numerical Methods, John Wiley \& Sons, New York 1966.

[16] S. P. Talwar, NASA Tech. Note D-2218, 1965. 